\title{
Optimization of Educational Systems Using Knapsack Problem
}

\author{
Nasim Zandi Atashbar and Fahimeh Rahimi
}

\begin{abstract}
The problem of automatically integrating adaptive content into different courses and curricula, thus exploiting the potential of the Internet in education is important. Learners' knowledge level is approached through a qualitative model of the level of performance that learners exhibit with respect to the concepts which are studied and are used to adapt the lesson contents and the navigation support. Learners' individual traits and especially their learning style represent the way learners perceive and process information, and are exploited to adapt the presentation of the educational material of a lesson. This paper proposes a new method to help learners find educational contents more adapted to their personalities in an efficient manner.
\end{abstract}

Index Terms-Adaptive learning, Bayesian networks, 0/1 Knapsack, Branch and bound algorithm, Sequencing, Elearning.

\section{INTRODUCTION}

Content of student books should reflect their needs and society's needs. Current instruction material however is uniform throughout each grade without focus on individual trait's or group differences. This problem makes educational materials do not appeal for some students.

Establishing a progressive trend in compiling student books is an effective way of meeting the student's needs and improving the instruction efficiency. Continuous scientific growth of societies and expansion of knowledge as well as information has created many new technologies in many countries. For instance, E-learning has been met with enthusiasm in many teaching levels. Here, we have offered a solution according to which selection of teaching material will be a progressive process and content of books will be different for students, reflecting their cultural and local needs as well as their individual traits such as their level of intellectual growth and intelligence.

The article is organized as follows. In the next section, find out learner's features by Bayesian networks. Then It tries to select the most appropriate adaptive learning objects with 0/1 knapsack problem in a limited amount of time determined by learner, section 3 , branch and bound algorithm is proposed to solve 0/1 knapsack problem efficiently. Finally, selected learning objects are sequenced in order to preserve the prerequisite.

Manuscript received May, 30, 2012; revised September 25, 2012

The authors are with Isfahan Mathematics House, Isfahan, Iran (email:nasim.zandi@yahoo.com; f_rahimi913@yahoo.com)

\section{RELATED WORKS}

When we use the word "Adaptive", we want to emphasize on the ability of change in different situations .One of the features of the adaptive system is that highlight learner's preferences, interests and browsing behaviors to offer personalized services [1]. However, the educational content must be suitable for the particular requirements of each individual, they have to be flexible enough to be used by a wide range of community of developers and learners [2]. In the last decade, a wide variety of researches have been done on this issue. Course Maintenance System, Adaptive Courseware Generation and Dynamic Courseware are the three main approaches [3] that have been proposed on this case and numerous systems have been developed based on them. Martinez and Bunderson introduced learner-difference profiles called learning orientations and the System for Intentional Learning and Performance Assessment (SILPA), which an interactive Web learning environment, to investigate how individuals manage learning in environments design to support learning orientations. In 2004,Lo,Wang and Yeh described the development of Hypermedia-based English Learning system for Prepositions (HELP), which provides EFL students learning diagnosis and remedial instruction according to student confidence scores (CR). Chen and Zhang select the learning content based on learner's cognitive state and present learning content through selecting teaching media based on learner's learning style. However, despite the existence of these methods, we need to create the enough flexible approach to accommodate the learner's needs.

\section{Methodology}

There are different categories of content in student books such as texts, examples, exercises and etc which these are called elements. A certain portion of the time allocated for teaching a book is set aside for each one of these elements. First, students take the standard test and their rate of learning is measured. Then, the result of this test is changed into a coefficient. The time needed for teaching each of the above mentioned elements are calculated by multiplying the original time allocated for each element by this coefficient. We can calculate time indeed for all of elements. This time called $w_{i}$ for element $i$-th. calculated in this way is expressed as the importance of each above categories is subjectively determined by the designer of the teaching as well as the time allocated for teaching the entire book as constraints. The decision can be made in more objective manners. We have used the same constraints in solving the knapsack problem.

Currently, there is an acute need for content which meets individual learner's needs. Local and regional conditions 
should be studied carefully before such content can be designed effectively. These conditions are expressed as factors which can be determinant of individuals and elements of the content which is considered. These factors are designated here as table 1 shows some factors and their possible values.

TABLE I: FACTORS AND THEIR POSSIBLE VALUES

\begin{tabular}{|c|c|}
\hline Quantity & Factors \\
\hline Boy-girl & Sex \\
\hline & Rate of hardship \\
\hline City-country & Location \\
\hline
\end{tabular}

Each of these factors may effect on some elements but not all of them. Nevertheless, we have defined a matrix $1 \times n$ where keep the amount of these factors for that specific element. In combination with the environmental and cultural conditions expressed as the $n$ factors. Another matrix can be written for the same factors but in combination with an individual student the degree of importance of each element for a student can be expressed as the number of factors which have the same value in two matrices. This number is designated for $i$-th element.

\section{A. Adaptive Learning}

The goal of this paper is to select the elements which match with the learner's characteristics and features most. So we need to obtain learner's features and store them in a suitable structure (learner model) for further use. But one of the difficulties we face with is that these modeling tasks involve a high level of uncertainty [7]. In order to overcome this issue, $[7,8]$ has proposed a method that we use it in our paper. This method introduces how to use Bayesian networks (BN) as a unifying framework to manage uncertainty in learner modeling. We pose various questions to find out the learner's characteristics and features .These questions explicitly have been asked from learner. Another way is to recognize them in an implicit manner, considering the behavior of the learner in our educational environment. In the following we explain how Bayesian network works.

\section{B. Bayesian Networks}

Bayesian network is a combination of two definitions of mathematical area: probability theory and graph theory. The graphical model of Bayesian network is using directed acyclic graph (DAG). The nodes in $\mathrm{BN}$ represent propositional variables of interest. The links of a BN represent informational or casual dependencies among the variables. These dependencies are quantified by conditional probabilities for each node given its parental nodes in the DAG. It allows us to compute the probability of a state of the variable given the state of its parent [9]. Fig. 1(a) shows a simple Bayesian network.

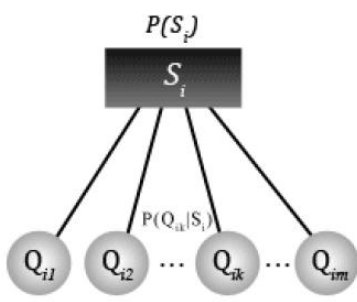

(a)

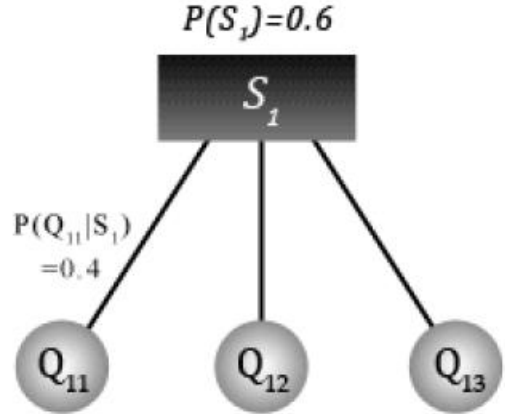

(b)

Fig. 1. (a) Diagram of the structure of a BN. (b) A sample of a BN.

Bayesian network is based on the Bayes theory which is shown in (1).

$$
p(a \mid b)=\frac{p(a, b)}{p(b)}=\frac{p(a) p(b \mid a)}{p(b)}
$$

With the help of Bayesian networks, we make a learner model to show the relation between learner's characteristics and questions. In our method, each learner's feature has its own BN. For example, in Figure 1(a), $p\left(s_{i}\right)$ represents the presence probability of feature $s_{i}$ in learner. These probabilities can be obtained from the statistics achieved by psychologists. Also we can use these statistics to indicate that, if the learner has the feature $s_{i}$, with which probability he/she can answer the question $Q_{i k}$. In this Bayesian network $p\left\langle Q_{i k} \mid s_{i}\right\rangle$ shows this probability $(1<k<m)$. After evaluating answers of the learner to each relevant question, $p\left(s_{i}\right)$ will be updated by following:

- If the answer was correct, $p\left(s_{i}\right)$ will be increased.

- If the answer was incorrect, $p\left(s_{i}\right)$ will be decreased.

For example, in Figure 1(b), $p\left(s_{i}\right)=0.6$. If the learner answers $Q_{11}$ were correct, the probability of having this feature will be updated by following:

As we see, $p\left(s_{i}\right)$ increased, but what would be the result if the learner selects the wrong answer to the question? By calculating the probability of $p\left(s_{i} \mid Q_{11}=\right.$ false $)$, the result is 0.529 . It is obvious that, $p\left(s_{i}\right)$ has been decreased. We do this process for every question related to this feature in order to obtain the final result.

Finally, we create a binary matrix $1 \times n$ for each element to store the element's features. Each column in this matrix represents a feature and entries of the matrix are only 0 or 1. If the value of $i$-th entry is one, it indicates that this element has $i$-th feature. After evaluating the learner's answers and obtaining the learner's features, we create another $1 \times n$ matrix to store learner's features. These matrices are illustrated in Figure 2. Now by comparing each element 
matrix with learner's feature matrix, we can obtain the position of that element with the learner's features. Adding the entries which their value is one in both matrices forms a variable which indicates the profit of $i$-th element for the learner.

\begin{tabular}{|c|c|c|c|c|c|c|c|}
\hline$k_{2}$ & $k_{z}$ & $k_{3}$ & & & $k_{i}$ & & $k_{s}$ \\
\hline 1 & $o$ & $o$ & 1 & $\cdots$ & 1 & 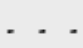 & $o$ \\
\hline \multicolumn{8}{|c|}{ (a) } \\
\hline$k_{2}$ & $k_{z}$ & $k_{3}$ & & & $k_{\dot{i}}$ & & $k_{n}$ \\
\hline$o$ & $O$ & 1 & 1 & $\cdots$ & 1 & $\because$ & 1 \\
\hline
\end{tabular}

Fig. 2. (a) Elements' feature matrix (b) Learner's feature matrix

\section{Selecting Appropriate Elements}

In order to present the most suitable teaching materials to the learner in a limited period of time, we have applied $0 / 1$ Knapsack problem. The 0/1 knapsack problem is one of the most famous combinatorial optimization problems. It is known as a classical NP-hard problem which has a large search space $[10,11,12]$. The $0 / 1$ knapsack problem can be stated briefly as: given a finite number of objects and a knapsack, find the maximum total profit under the constraint that the total weight of all chosen objects is at most the weight limit [13,14,15].

\section{The formulation of the 0/1 Knapsack Problem}

The 0/1 knapsack problem (KP) is defined exactly as follows: We are given $n$ elements and a knapsack. Element $i$ has a weight $w_{i}$ and the knapsack has a weight limit $\mathrm{C}$. If object $i$ is placed into the knapsack, we will obtain a profit $p_{i}$. The problem is to maximize the total profit under the constraint that the total weights of all chosen objects are at most C. So, the knapsack problem can be formulated as $[16,14,17,18]$ :

$$
\begin{array}{ll}
\text { maximize } & z=\sum_{j=1}^{n} p_{j} x_{j} \\
\text { subject to } & \sum_{j=1}^{n} w_{j} x_{j} \leqslant c, \\
& x_{j} \in\{0,1\}, \quad j \in\{1, \ldots, n\} .
\end{array}
$$

where $x_{i}$ is a binary variable denoting whether object $i$ is chosen or not. Equation (3) is the constraint that needs to be satisfied and defines the profit of a feasible n-tuple. Figure 3 demonstrates our problem.

\section{E. Modeling Proposed Method to 0/1 Knapsack}

Now we try to model our element selection method for a 0/1 knapsack as follows:

Because of time constraints, learner determines his/her available time. This value is the total amount of time available to our teaching process. Each element group has an important factor which is defined by the author of educational content. We can calculate the time needed for each element group by considering this factor and total amount of available time. Obtained time is the constraint value of the knapsack and we define it as $c_{j}$ indicates the $\mathrm{j}$ th element group. Each element has its own importance factor. So the author must assign a pre-defined time needed to be studied by learner to each element.

On the other hand learning speed and performance is not equal in every learner. So we need to obtain the speed of the learner in comprehension of educational contents. To achieve learner's speed factor, we use available standard assessments (e.g. Stanford-Binettest). The results of this assessment are transformed to a coefficient. By multiplying this coefficient to each element's minimum time we can attain the time needed to study that element. Each element's time amount is denoted by $w_{i}$ which $i$ indicates the $i$-th element.

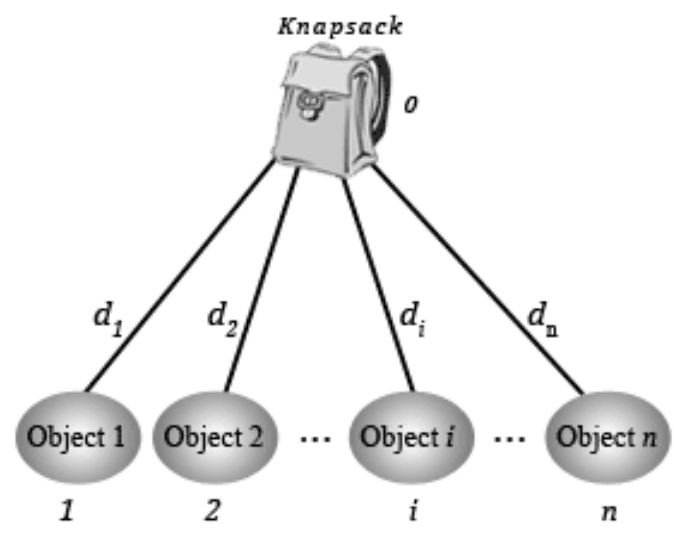

Fig. 3. The description of a 0/1 knapsack problem

\section{F. Basic Branch-And-Bound Algorithms}

Algorithms for KPs are mainly based on approach: branch-and-bound. The actual performance of an algorithm, however strictly depends on the way tight upper bounds are applied.

The first upper bound for KP, based on a continuous relaxation, was presented by Dantzig in mid 1950s. It is obtained by sorting the items so that

$$
\frac{p_{j}}{w_{j}} \geqslant \frac{p_{j+1}}{w_{j+1}} \quad(j=1, \ldots, n-1)
$$

and determining the critical variable through

$$
s:=\min \left\{i: \sum_{j=1}^{i} w_{j}>c\right\} \text {. }
$$

The continuous upper bound for KP is then

$$
\left\lfloor\sum_{j=1}^{s-1} p_{j}+\left(c-\sum_{j=1}^{s-1} w_{j}\right) \frac{p_{s}}{w_{s}}\right\rfloor
$$

and the corresponding Dantzig integer solution is

$$
j<s \text { and } x_{j}=0 \text { for } j \geq s .
$$

No better bounds were presented in the following two decades until Martello and Toth presented a tighter bound by imposing integrality on the critical variable. Since then, several other bounds have been presented based on Lagrangian relaxation, partial enumeration, construction of 
valid additional constraints, and different relaxations of the latter .The first branch-and-bound algorithms for KP appeared in the early 1970s. Among the most successful we should mention the algorithms by Horowitz and Sahni, Fayard and Plateau, Nauss and Martello and Toth. These algorithms are all based on a depth-first enumeration in order to limit the space consumption. The branching strategy consists of selecting an item $\mathrm{j}$ and generating two children nodes through conditions $\mathrm{xk}=0$. Upper bounds for these algorithms are derived from some kind of continuous relaxation of the currently induced sub problem.

A comparison of the algorithms, presented and Toth, shows that they perform well for small sized "easy" instances [18].

\section{APPLICATION}

Each learner has his/her own account and individual profile. As entering the application for the first time, each learner faces with a bunch of questions in order to attain the learner's personalities, characteristics and learning styles. According to learner's answers the Bayesian networks will be updated.

\section{CONCLUSION AND FUTURE WORKS}

Using adaptive learning in learning environments is one useful approach in education systems. The problem is that learner faces with a massive amount of contents during the learning process while he/she has a limited time. As a result, he/she may feel frustrated and disappointed. In this paper we tried to overcome this issue and produce an optimized educational content which satisfies the learner's actual needs by considering his/her personalities and features in a limited amount of time. A tutoring application has been developed by authors of this paper to provide a practical approach to our method. Our application which is based on our proposed method showed that it has a great impact on adaptive learning.

The future work will focus on optimizing the learner's model to be synchronous with learner's behaviors sudden changes. Also we will try to create a fully integrated webbased learning system based on our method.

\section{REFERENCES}

[1] C. Chen, "Personalized E-Learning System with Self-Regulated Learning Assisted Mechanisms for Promoting Learning Performance," Expert Systems with Applications, vol. 36, pp. 8816-8829, 2009.

[2] P. Brusilovsky and J. Vassileva, "Course sequencing techniques for large scale web-based education," International Journal of Continental Engineering Education and Lifelong Learning, vol. 13 , no. 1-2, pp. 75-94, 2003.

[3] M. Martinez and C. V. Bunderson, "Building interactive world wide web learning environments to match and support individual learning differences," Journal of Interactive Learning Research, vol. 11, no. 2, pp. 131-162, 2000.

[4] J. J. Lo, H. M. Wang, and S. W. Yeh, "Effects of confidence scores and remedial instruction on prepositions learning in adaptive hypermedia," Computers \& Education, vol. 42, no. 1, pp. 45-63, 2004.
[5] S. Chen and J. Zhang, "The Adaptive Learning System Based on Learning Style and Cognitive State," International Symposium on Knowledge Acquisition and Modeling, pp. 302- 306, 2008.

[6] C. Conati, A. Gertner, and K. Vanlehn, "Using bayesian networks to manage uncertainty in student modeling," User Modeling and UserAdapted Interaction, vol. 12, no. 4, pp. 371-417, November 2002.

[7] S. Kardan and A. Kardan, "Towards a More Accurate Knowledge Level Estimation," Third International Conference on Information Technology, pp. 1134-1139, 2009.

[8] S. Sanghai, "Relational Dynamic Bayesian Networks," Journal of Aritificial Intelligence Research, vol. 24, pp. 1-39, 2005.

[9] A. Jaszkiewicz, "Comparison of Local Search-based Metaheuristics on the Multiple Objective Knapsack Problem," Foundations of Computing and Decision Sciences, vol. 26, pp. 99-120, 2001.

[10] W. Shin, "A branch and bound method for the multiconstraint zeroone knapsack problem," Journal of the Operational Research Society, vol. 30, no. 4, pp. 369-378, April 1979.

[11] M. Garey and D. Johnson, Computers and Intractability: A guide to the Theory of NP-Completeness. San Francisco: W. H. Freeman \& Co, 1979.

[12] A. Jaszakiewicz, "On the Performance of Multiple Objective Genetic Local Search on the 0/1 Knapsack Problem-A Comparative Experiment," IEEE Trans. on Evolutionary Computation, vol. 6, pp 402- 412, 2002.

[13] A. Vreville and G. Plateau, "Heuristics and reduction methods for multiple constraints 0- 1 linear programming problems," European Journal of Operational Research, vol. 24, pp. 206-215, 1986.

[14] S. Sahni, "Approximate algorithms for the 0-1 kanpsack problem," Journal of ACM, vol. 22, pp. 115- 224, 1975.

[15] E. Balas and E. Zemel, "Solving large zero-one knapsack problems," Operation Research, vol. 28, pp. 1130-1154, 1980.

[16] B. Gavish and H. Pirkul, "Efficient algorithms for solvingmulticonstraint zero-one knapsack problems to optimality," Maht. Programming, vol. 31, pp. 78-105, 1985.

[17] S. Martello, P. David, and P. Toth, "New trends in exact algorithms for the 0-1 knapsack problem," European Journal of Operational Research 123, pp. 325-332, 2000.

[18] A. Mazaheri, E. Haghshenas, A. Gholipour, M. Tavakoli, H. Narimani, N. Zandi, F. Rahimi, Sh. Nouri, "Introducing a new intelligent adaptive learning content generation method," Second International Conference on E-Learning and E-Teaching, 2010.

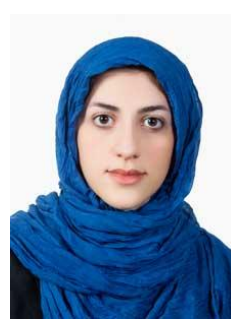

Nasim Zandi Atashbar was born in 9 Aug 1989 in Isfahan, Iran. She got Diploma in Mathematic science from National Organization For Development Of Exceptional Talent .She received Bachelor of Science in Industrial Engineering from Isfahan University of Technology in 2011.she has published several papers in field of Knapsack problem, Mathematics Education, Geographic Information System ,etc. She worked as Project planning and Controlling Engineering in Foolad Technic International Engineering Company. She researches and sets the workshops in Isfahan Mathematics House.

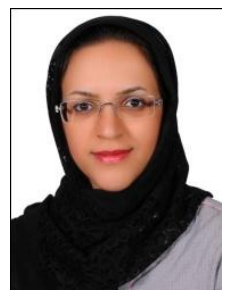

Fahimeh Rahimi was born in 4 Dec 1988 in Isfahan ,Iran. She got Diploma in Mathematic science from National Organization For Development Of Exceptional Talent. She received Bachelor of Science in Mathematic from Isfahan University of Thechnology in 2011. She is currently studing MS coursing in Mathematic at Isfahan University Of Thechnology. She has published several papers in field of Knapsack problem, Mathematics Education, etc .She researches and teaches Mathematic and sets the workshops in Isfahan Mathematics House. 